\title{
Südost- Forschungen
}

INTERNATIONALE ZEITSCHRIFT FÜR

GESCHICHTE, KULTUR UND LANDESKUNDE SÜDOSTEUROPAS

IM AUFTRAG DES

LEIBNIZ-INSTITUTS FÜR OST- UND SÜDOSTEUROPAFORSCHUNG

GELEITET UND HERAUSGEGEBEN VON

\section{ULF BRUNNBAUER UND KONRAD CLEWING}

IN VERBINDUNG MIT

Neven Budak (Zagreb), Marie-Janine Calic (München), Holly Case (Providence/RI), Nathalie Clayer (Paris), Basil Gounaris (Thessaloniki), Hannes Grandits (Berlin), Mehmet Hacısalihoğlu (Istanbul), Edgar Hösch (Würzburg), Christina Koulouri (Athen), Hans Georg Majer (München), Diana Miškova (Sofia), Günter Prinzing (Mainz), Joachim von Puttkamer (Jena), Milan Ristović (Belgrad), Oliver Jens Schmitt (Wien), Flavius Solomon (laşi), Christian Voß (Berlin)

Redaktion: PETER MARIO KREUTER

Band 75 2016

DE GRUYTER OLDENBOURG 


\section{Südost-Forschungen}

\section{Internationale Zeitschrift für Geschichte, Kultur und Landeskunde Südosteuropas}

Die jährlich erscheinende Zeitschrift ist ab Band 11 (1946/52) über den Verlag De Gruyter Oldenbourg, History, Rosenheimer Str. 143, D-81671 München, zu beziehen. Preis pro Band 122,- € zzgl. Versand kosten. Indices zu den Bänden 1 (1936) - 62 (2003) in: Südost-Institut München 1930-2005. München 2005, oder auf www.ios-regensburg.de/hoesch.pdf.

Beiträge sowie alle die „Südost-Forschungen“ betreffenden Besprechungsstücke, Anfragen und Mitteilungen sind zu senden an die Redaktion der Südost-Forschungen:

Dr. Peter Mario Kreuter, Leibniz-Institut für Ost- und Südosteuropaforschung, Landshuter Straße 4, D-93047 Regensburg, E-Mail: kreuter@ios-regensburg.de, Tel. + 49/941/9435472. Für redaktionelle Hinweise siehe unter http://www.ios-regensburg.de/fileadmin/dokumente/manuskriptgestaltung.pdf. Redaktionsassistenz: Frederik Lange.

(C) 2017 by Leibniz-Institut für Ost- und Südosteuropaforschung

Veröffentlicht durch Verlag De Gruyter Oldenbourg, München

Die Zeitschrift und alle in ihr enthaltenen Beiträge und Abbildungen sind urheberrechtlich geschützt. Mit Ausnahme der gesetzlich zugelassenen Fälle ist eine Verwertung ohne Einwilligung des Verlages strafbar.

Satz: Kraus PrePrint, Landsberg am Lech

Druck: Friedrich Pustet GmbH \& Co. KG, Regensburg

Printed in Germany

ISSN 0081-9077 